\title{
TOP1MT deficiency promotes GC invasion and migration via the enhancements of LDHA expression and aerobic glycolysis
}

\author{
Hongqiang Wang1,2, Rui Zhou', Li Sun', Jianling Xia', Xuchun Yang'2, Changqie Pan', \\ Na Huang', Min Shi', Jianping Bin33, Yulin Liao³ and Wangjun Liao' \\ 'Department of Oncology, Nanfang Hospital, Southern Medical University, Guangzhou, China \\ 2Department of Oncology, Zhoushan Hospital, Zhoushan, China \\ ${ }^{3}$ Department of Cardiology, Nanfang Hospital, Southern Medical University, Guangzhou, China
}

Correspondence should be addressed to W Liao

Email

nfyyliaowj@163.com

\begin{abstract}
Aerobic glycolysis plays an important role in cancer progression. New target genes regulating cancer aerobic glycolysis must be explored to improve patient prognosis. Mitochondrial topoisomerase I (TOP1MT) deficiency suppresses glucose oxidative metabolism but enhances glycolysis in normal cells. Here, we examined the role of TOP1MT in gastric cancer (GC) and attempted to determine the underlying mechanism. Using in vitro and in vivo experiments and analyzing the clinicopathological characteristics of patients with GC, we found that TOP1MT expression was lower in GC samples than in adjacent nonmalignant tissues. TOP1MT knockdown significantly promoted GC migration and invasion in vitro and in vivo. Importantly, TOP1MT silencing increased glucose consumption, lactate production, glucose transporter 1 expression and the epithelial-mesenchymal transition (EMT) in GC. Additionally, regulation of glucose metabolism induced by TOP1MT was significantly associated with lactate dehydrogenase A (LDHA) expression. A retrospective analysis of clinical data from 295 patients with GC demonstrated that low TOP1MT expression was associated with lymph node metastasis, recurrence and high mortality rates. TOP1MT deficiency enhanced glucose aerobic glycolysis by stimulating LDHA to promote GC progression.
\end{abstract} Key Words

- glycolysis

- gastric cancer

- lactate dehydrogenase A

- mitochondrial topoisomerase I

\section{Introduction}

Metabolic disturbance is a universal property of cancer cells and one of the most important causes of tumorigenesis and cancer progression (Hsu \& Sabatini 2008, Kroemer \& Pouyssegur 2008, Lu et al. 2015). The main metabolismrelated characteristic of most cancer cells is a metabolic switch from the mitochondrial tricarboxylic acid (TCA) cycle to aerobic glycolysis. Even under aerobic conditions, glucose is taken up and transformed to lactate via the glycolytic pathway in cancer cells, a phenomenon known as the Warburg effect (Warburg 1956). This metabolic switch produces an acidic microenvironment for the tumor and promotes the epithelial-mesenchymal transition (EMT) and metastasis, leading to poor prognosis (Vander Heiden et al. 2009, Hirschhaeuser et al. 2011, 
Chen 2012, Lu et al. 2015). Studies of the Warburg effect in tumors have been a major focus of tumor metabolism research. Therefore, analysis of the Warburg effect and exploring the underlying target genes may provide clues for therapeutic strategies in clinical oncology.

Mitochondrial topoisomerase I (TOP1MT) encodes a crucial mitochondrial DNA topoisomerase that plays an important role in relieving mitochondrial DNA tension and supercoiling produced during replication and transcription (Zhang et al. 2001). TOP1MT deficiency influences mitochondrial DNA expression, which decreases the expression of genes in the oxidative phosphorylation (OXPHOS) system involved in both OXPHOS and electron transport in normal cells (DiMauro \& Schon 1998, Zhang et al. 2001). Additionally, defective TOP1MT gene expression may predispose individuals to various diseases related to mitochondrial disorders, e.g., diabetes, Alzheimer's disease and Parkinson's syndrome. Interestingly, TOP1MT is involved in mitochondrial OXPHOS not only in normal cells but also in cancer cells (Goto et al. 2006). However, the role of TOP1MT in cancer cells has not been reported.

In normal cells, the mechanism through which TOP1MT affects metabolism involves enhancement of OXPHOS (Douarre et al. 2012). TOP1MT deficiency leads to mitochondrial respiratory chain defects, which inhibit the mitochondrial TCA cycle and OXPHOS. However, through alternative pathways, cells with TOP1MT deficiency enhance glycolysis for energy production. In this process, the TCA cycle and OXPHOS decrease, glucose consumption and lactate production increase and glycolytic enzymes are concomitantly activated. For example, lactate dehydrogenase A (LDHA) is a glycolytic enzyme that catalyzes the formation of lactate from pyruvate. Even in cancer cells, LDHA plays important role in controlling the speed of glycolysis and maintaining the continuity of aerobic glycolysis (Fantin et al. 2006, Ganapathy et al. 2009). Studies have demonstrated that aerobic glycolysis promotes cancer cell progression (Chen 2012, Lin et al. 2015, Lu et al. 2015), and mitochondrial OXPHOS suppresses tumor metastasis (Lu et al. 2015); thus, metabolic therapies are potential therapeutic strategies for prevention or intervention of cancer metastasis (Kolev et al. 2008, Le et al. 2010, Fiume et al. 2014, Augoff et al. 2015). In fact, studies on tumor metabolism have demonstrated that knowledge of tumor glycolysis may facilitate the discovery and development of therapeutic strategies for clinical oncology (Giatromanolaki et al. 2006, Koukourakis et al.
2006, 2009, Chen 2012). Moreover, analysis of TOP1MT may provide clues for therapeutic strategies by regulating the Warburg effect in tumors.

Therefore, in this study, we sought to determine the role of TOP1MT in invasion and migration and elucidate the regulatory metabolic mechanisms through which TOP1MT is involved in GC. We discovered that TOP1MT deficiency promoted GC cell invasion and migration; increased glucose consumption, lactate production and LDHA activity in vitro and in vivo and promoted tumor metastasis in patients with GC. This study is the first report demonstrating the roles of TOP1MT in GC and the mechanisms through which TOP1MT regulates the Warburg effect to promote GC progression.

\section{Materials and methods}

\section{Patients and tissue samples}

All patients enrolled in this study provided informed consent, and the Nanfang Hospital Ethics Review Board approved our research. Fresh tissue specimens were obtained from 12 patients who underwent radical surgery for GC between May and June 2015. Paraffinembedded tissue specimens for immunohistochemistry were obtained from 295 patients with GC who had not undergone any neoadjuvant chemotherapy before surgery in our hospital between 2006 and 2011.

\section{Real-time quantitative reverse transcription polymerase chain reaction (RT-PCR)}

Total RNAs were acquired from GC specimens and cell lines using TRIzol reagent and reverse transcribed with M-MLV reverse transcriptase. Real-time PCR was conducted using a SYBR Green I Master kit on a LightCycler 480 system. $\beta$-Actin expression was determined as an internal control. The primer sequences for real-time RT-PCR are shown in Supplementary Table 1 (see section on supplementary data given at the end of this article).

\section{Western blotting}

Cells were lysed in cell lysis buffer. Equal amounts of proteins from cell extracts were resolved by sodium dodecyl sulfate polyacrylamide gel electrophoresis on $10 \%$ gels and transferred to polyvinylidene difluoride membranes (Millipore). The blots were blocked in a solution of $10 \%$ skimmed milk and incubated with primary 
antibodies overnight at $4^{\circ} \mathrm{C}$. Subsequently, the blots were washed and incubated with secondary antibodies for $1 \mathrm{~h}$. Antibody-bound protein bands were detected using an Odyssey Infrared Imaging System (LI-COR Biosciences, Lincoln, NE, USA).

\section{Immunohistochemistry}

Immunohistochemical staining was performed according to a published standard protocol (Wang et al. 2013, Lin et al. 2015, Xia et al. 2016). The staining intensity was scored as 0 (no staining), 1 (weak staining), 2 (medium staining) or 3 (strong staining). Staining extent was scored based on the percentage of positive cells as $0(0 \%), 1$ (1\%-25\%), 2 (26\%-50\%), $3(51 \%-75 \%)$ or 4 (76\%-100\%). The final staining scores (0-12) for TOP1MT and LDHA expression were obtained by multiplying the intensity and the extent scores. Tumor tissues with final staining scores of less than 6 and greater than or equal to 6 were regarded as having low and high TOP1MT expression, respectively.

\section{Methyl thiazolyl tetrazolium (MTT) assay}

Cell proliferation was measured using MTT assays. Briefly, BGC-823 or SGC-7901 cells were seeded into 96-well plates at a density of $4 \times 10^{3}$ cells/well. On the indicated days after plating, $200 \mu \mathrm{L}$ MTT solution $(5 \mathrm{mg} / \mathrm{mL})$ was added to each well at $0,24,48,72$ and $96 \mathrm{~h}$. The samples were then incubated for $4 \mathrm{~h}$ at $37^{\circ} \mathrm{C}$. Subsequently, the medium was replaced with $150 \mu \mathrm{L}$ dimethyl sulfoxide for an additional 10-min incubation. The absorbance was then determined at $570 \mathrm{~nm}$ with a microplate reader (Molecular Devices).

\section{Colony formation assay}

A total of 200 GC cells were seeded onto 6-well culture dishes. After 14 days of culture, the cells were fixed with 4\% paraformaldehyde and stained with Giemsa solution. Colonies containing at least 50 cells in each well were counted using the following formula: plate clone formation efficiency $=$ (number of colonies/number of cells inoculated) $\times 100 \%$.

\section{Wound healing assay}

Cells were plated in 6-well plates at a density of $5 \times 10^{4}$ cells/ well. After cells reached confluence, they were scratched using a sterile plastic tip. Wound closure was visualized at $\mathrm{Oh}$ and $24 \mathrm{~h}$ using an inverted microscope.

\section{Transwell migration assay}

Cells $\left(1 \times 10^{5} / \mathrm{mL}, 200 \mu \mathrm{L}\right)$ in RPMI-1640 (Invitrogen, Life Technologies) without serum were plated in the upper chambers of wells containing 8 - $\mu \mathrm{m}$ pore size polycarbonate filters (Costar, Bodenheim, Germany). The lower chamber was filled with $600 \mu \mathrm{L}$ RPMI-1640 containing 10\% fetal bovine serum (FBS). Cells were incubated for $24 \mathrm{~h}$ at $37^{\circ} \mathrm{C}$. The upper chambers were washed twice. Cells invading through chambers and adhering to the lower surface were fixed with 4\% paraformaldehyde for $15 \mathrm{~min}$ and stained with hematoxylin for $20 \mathrm{~min}$. The invading cells on the lower surface of the chamber were photographed and counted under a microscope in five randomly selected fields.

\section{Boyden chamber invasion assays}

Cell invasion assays were similar to transwell migration assays, except that the polycarbonate filters of the upper chamber were coated with Matrigel (BD Biosciences).

\section{Knockdown of target genes by small interfering RNAs (siRNAs)}

Transfection of BGC-823 and SGC-7901 cells with siRNAs was performed using Lipofectamine 2000. For transient transfection, cells were transfected with siRNAs for $6 \mathrm{~h}$ and then cultured in RPMI-1640 with 10\% FBS for $48 \mathrm{~h}$ before subsequent functional assays. The sequences of siRNAs for TOP1MT and LDHA are listed in Supplementary Table 2. Cells treated with the transfection reagents alone were included as negative controls.

\section{Glucose consumption, lactate production, pH and LDHA activity assays}

As in our previous study (Lin et al. 2015), $1 \times 10^{5}$ cells were seeded in each well of 6-well plates and incubated under normoxic conditions for $24 \mathrm{~h}$ at $37^{\circ} \mathrm{C}$. The culture medium of cells was collected for lactate, glucose and $\mathrm{pH}$ assays. Lactate and glucose concentrations were measured using enzymatic methods (Randox, Crumlin, Antrim, UK). pH values were determined using a $\mathrm{pH}$ meter. Cytoplasmic LDHA activity was measured with assay kits (Comin, Suzhou, China). 


\section{ATP determination}

Cellular ATP generation was determined using an ATP determination kit based on firefly luciferase (Beyotime, Haimen, China).

\section{Cell culture and stable cell lines}

Cell culture and stable cell line establishment were performed as previously described (Wang et al. 2013). The human gastric epithelial cell line GES-1 and GC cell lines BGC-823, AGS, MKN-7, SGC-7901, MKN-28, MKN-45 and MGC-803 were purchased from Foleibao Biotechnology Development Company (Shanghai, China). Molecular authentication of each cell line was performed by short tandem repeat analysis, and passage numbers used for the experiments are provided (Supplementary Fig. 1). Cells were cultured in RPMI-1640 with $10 \%$ FBS at $37^{\circ} \mathrm{C}$ in $5 \% \mathrm{CO}_{2}$. Sodium oxamate, an LDHA inhibitor, was obtained from Sigma-Aldrich. In the in vivo assay, cells exhibiting stable TOP1MT knockdown were established by transfection of BGC-823 and SGC-7901 cells with short hairpin RNA targeting TOP1MT (shTOP1MT) (Wang et al. 2013).

\section{Xenograft model}

The influence of TOP1MT on tumor metastasis was further demonstrated in vivo. All null nude mice were treated according to ethical guidelines for the care and use of experimental animals. shTOP1MT and scramble control $\left(5 \times 10^{6}\right)$ GC cells (BGC-823 and SGC-7901 cell lines) were subcutaneously injected into the flanks of 4 -week-old female null nude mice ( $n=40,10$ mice per cell line). Tumor sizes were evaluated every 4 days with a digital caliper. Tumor volumes were obtained as follows: volume $=$ width $\times$ length $\times($ width + length $) / 2$. On day 28 after inoculation, the mice were killed, and tumors were harvested and weighted. To test the influence of TOP1MT on tumor metastasis in vivo, null nude mice were subjected to caudal vein injection $\left(2 \times 10^{6}\right.$ cells/ mouse, $n=48,12$ mice per cell line) of BGC-823 and SGC7901 cells with shTOP1MT or scramble control shRNA. The molecular changes in cells with or without TOP1MT silencing were confirmed by Western blotting, and LDHA expression was checked by immunohistochemistry. On day 40 after caudal vein injection, the mice were killed and dissected. Pulmonary metastasis nodules were counted and visualized by hematoxylin and eosin staining.

\section{Statistical analysis}

Unless otherwise indicated, all assays were repeated at least three times, and representative results were reported as means \pm standard deviations (S.D.). Statistical significance was determined by Student's t-test or one-way analysis of variance (ANOVA) with Tukey's multiple comparison tests. Correlations between two continuous variables were examined by Pearson's test. Survival rates were analyzed by the Kaplan-Meier method and examined by log-rank tests. Differences with $P$ values of less than 0.05 were considered statistically significant. The SPSS13.0 software program was used for analyzing all data.

\section{Results}

\section{Decreased expression of TOP1MT in GC}

We first detected the RNA expression of TOP1MT in tissue by RT-PCR and found that TOP1MT mRNA levels were lower $(P<0.05)$ in $\mathrm{GC}$ tissues than in adjacent normal tissues (Fig. 1A). Western blotting results also showed that TOP1MT expression was significantly lower in GC tissue samples (Fig. 1B).

We further examined TOP1MT expression in seven human GC cell lines (BGC-823, AGS, MKN-7, SGC-7901, MKN-28, MKN-45 and MGC-803) and a normal gastric epithelial cell line (GES-1). We also found that TOP1MT expression was lower at both mRNA (Fig. 1C) and protein levels (Fig. 1D) in GC cell lines compared with that in GES-1 cells.

\section{Silencing of TOP1MT enhanced the migration and invasion of GC in vitro and in vivo}

Our initial results demonstrated that TOP1MT expression was low in both GC tissue samples and cell lines. However, it was still unclear whether this low TOP1MT expression affected the functions of GC cells. To explore the effects of low TOP1MT expression on GC cells, we performed functional experiments in BGC-823 and SGC-7901 cells. Cells were transiently transfected with siRNA targeting TOP1MT. Both siTOP1MT1 and siTOP1MT2 significantly inhibited TOP1MT mRNA (Supplementary Fig. 2A) and protein expression (Supplementary Fig. 2B and C). siTOP1MT1 was selected for the following functional experiments because of its higher inhibitory effect. To demonstrate that the shTOP1MT constructs specifically targeted the mitochondrial isoform of TOP1, mRNA and protein levels of TOP 1, TOP $2 \alpha$, TOP $2 \beta$, ТОР $3 \alpha$ and TOР $3 \beta$

Published by Bioscientifica Ltd. 
A

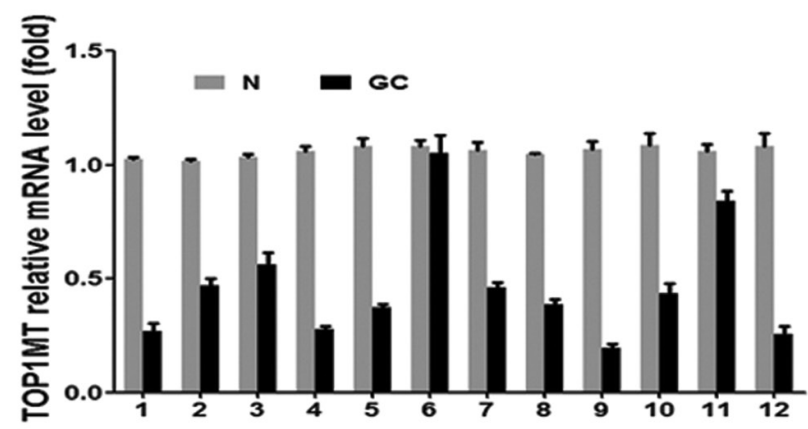

B

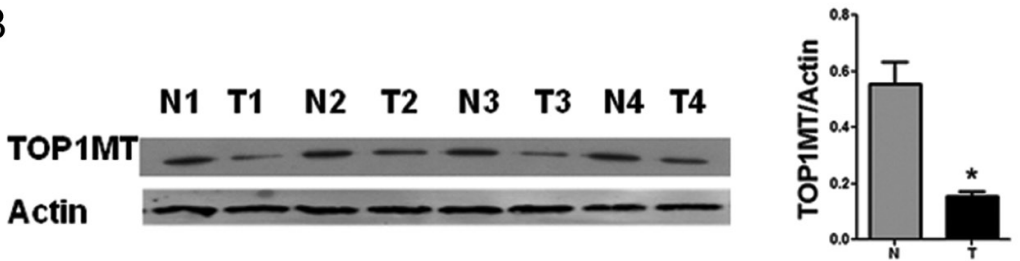

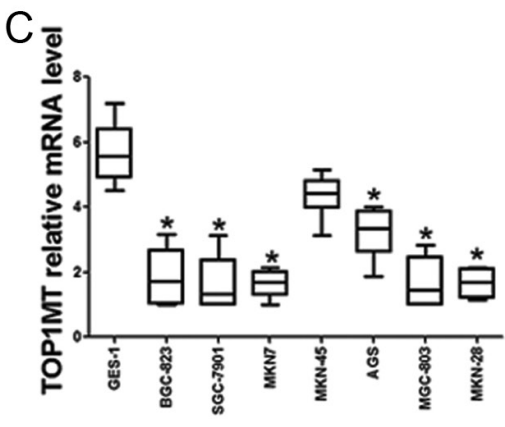

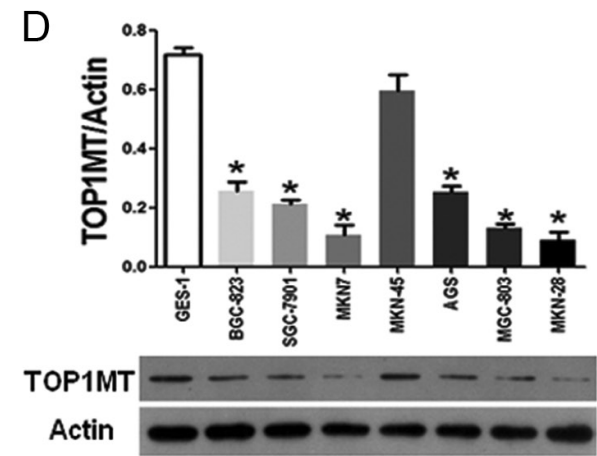

Figure 1

TOP1MT expression was downregulated in gastric cancer tissues and cell lines. (A and B) TOP1MT expression levels in gastric cancer (GC) tissues from patients and normal adjacent tissues (N) were analyzed by RT-PCR (A) and Western blotting (B). (C and D) TOP1MT expression levels in GC cell lines and GES-1 normal gastric epithelial cells were analyzed by RT-PCR (C) and Western blotting (D). ${ }^{*} P<0.05$ in comparisons of GC tissues (T) or GC cell lines with normal adjacent tissues

(N) groups or GES-1 normal gastric epithelial cells. were tested by RT- PCR and Western blotting. TOP1MT knockdown did not affect the mRNA or protein levels of these targets in GC cells (Supplementary Fig. 3C, D and $\mathrm{E})$.

Methyl thiazolyl tetrazolium (MTT) assays and colony formation assays showed that TOP1MT silencing failed to influence the proliferation of GC cells (Supplementary Fig. 3A and $\mathrm{B}$ ). However, in transwell assays, the numbers of migrating (Fig. 2A) and invading cells (Fig. 2B) were significantly higher for siRNA-transfected BGC-823 and SGC-7901 cells. In addition, wound-closure migration assays also showed that TOP1MT silencing significantly accelerated wound recovery (Fig. 2C).

Furthermore, to determine the effects of low TOP1MT expression in vivo, TOP1MT silenced (shTOP1MT) BGC823 and SGC-7901 cells and their corresponding negative controls, BGC-823/scramble and SGC-7901/scramble were established. BGC-823/shTOP1MT, BGC-823/ scramble, SGC-7901/shTOP1MT and SGC-7901/scramble cells were subcutaneously injected into 40 athymic female mice. The results showed that TOP1MT knockdown did not influence tumor growth (Fig. 2D). We assessed the metastasis of GC cells by injecting BGC-823/shTOP1MT, BGC-823/scramble, SGC-7901/shTOP1MT and SGC-7901/ scramble cells into the caudal vein of 48 athymic female mice. Again, TOP1MT knockdown increased pulmonary metastasis in both cell lines, as demonstrated by anatomical pathology and histopathology (Fig. 2E). These results clearly indicated that TOP1MT silencing promoted the invasion and metastasis of GC cells.

\section{Silencing of TOP1MT upregulated glycolysis in GC cells}

Given that TOP1MT deficiency was found to promote GC cell invasion and migration, we further explored the potential mechanisms. In normal cells, TOP1MT silencing has been found to enhance cell glycolysis (Douarre et al. 2012), and in cancer cells, glycolysis promotes cell metastasis (Chen 2012, Lin et al. 2015, Lu et al. 2015). Therefore, enhanced glycolysis induced by TOP1MT deficiency may be a key causal factor in promoting GC progression. We explored the impact of TOP1MT on glycolysis in GC cells. TOP1MT silencing significantly increased glucose utilization, lactate production and 
A
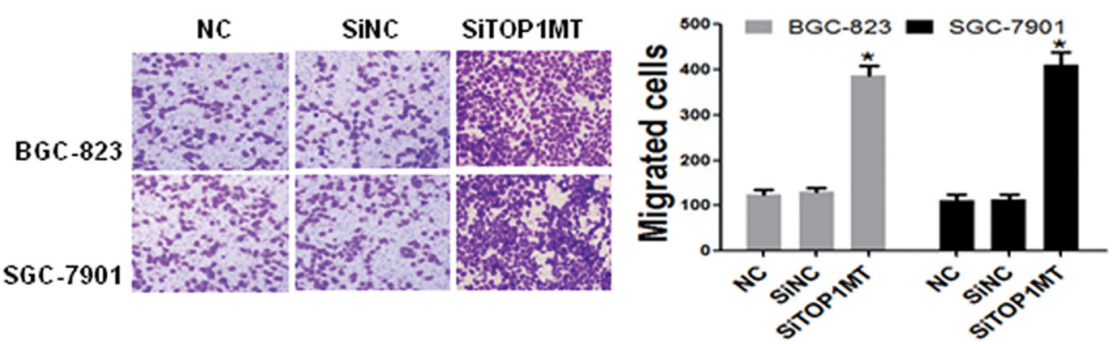

B
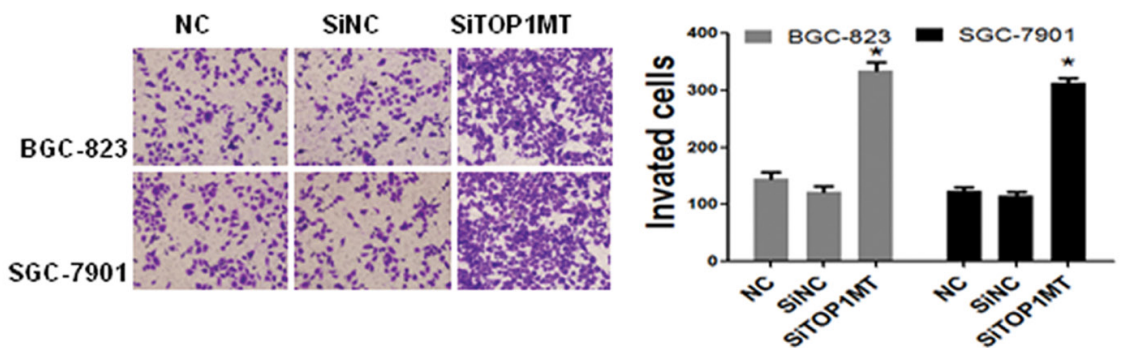

C

oh

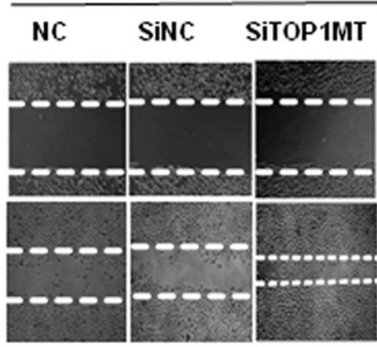

D

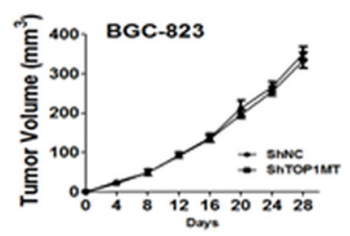

BGC-823

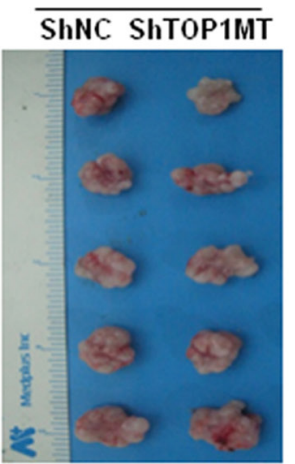

SGC-7901

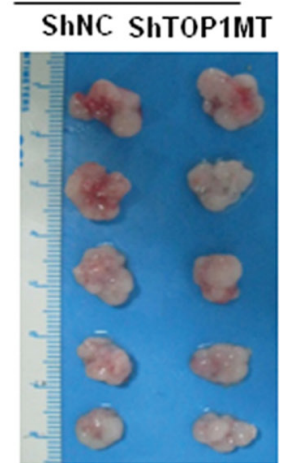

SGC-7901
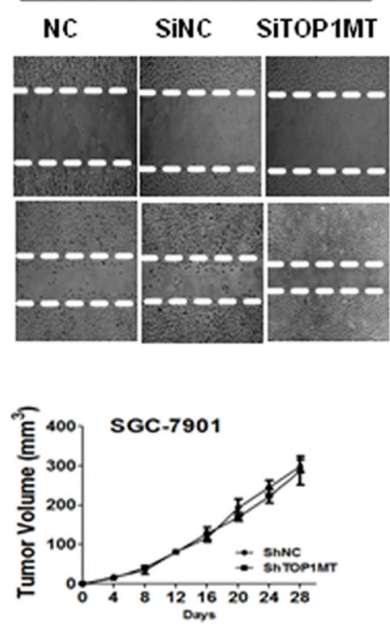

E
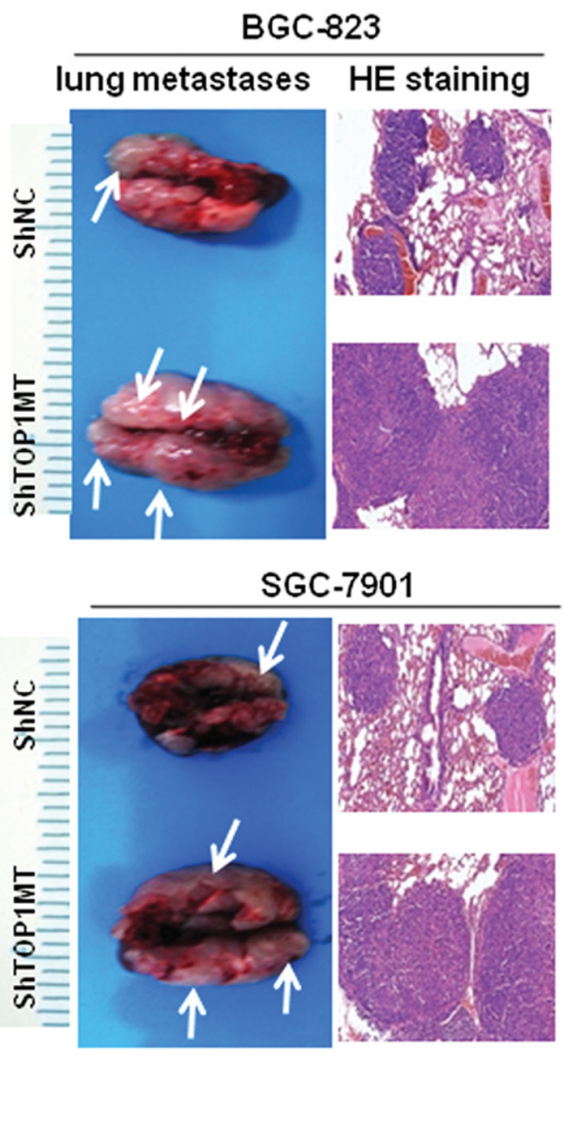

Figure 2

TOP1MT silencing enhanced the invasion and migration of BGC-823 and SGC-7901 cells in vitro and in vivo. (A, B and C) Migration and invasion of GC cells infected with TOP1MT siRNA (SiTOP1MT) or negative control siRNA (SiNC) or negative control (NC) were assessed by transwell migration assays (A), Boyden chamber invasion transwell assays (B), and wound closure assays (C). (D) BGC-823/scramble, BGC-823/shTOP1MT, SGC-7901/scramble, and SGC-7901/shTOP1MT were subcutaneously injected into the flanks of female athymic mice. The volumes (D, upper) of the xenotransplanted tumors were calculated every 4 days. Representative images of subcutaneous xenotransplanted tumors ( $D$, lower left) and tumor weights ( $D$, lower right) were measured when the mice were killed on day 28 post implantation. (E) Gross lung metastases ( $E$, left) and lung metastases stained with hematoxylin and eosin (HE staining) (E, right) for BGC-823 and SGC-7901 cells. * $P<0.05$ in comparisons of the SiTOP1MT-transfected group with the SiNC or NC group. A full colour version of this figure is available at http://dx.doi.org/10.1530/ERC-17-0058. 
A
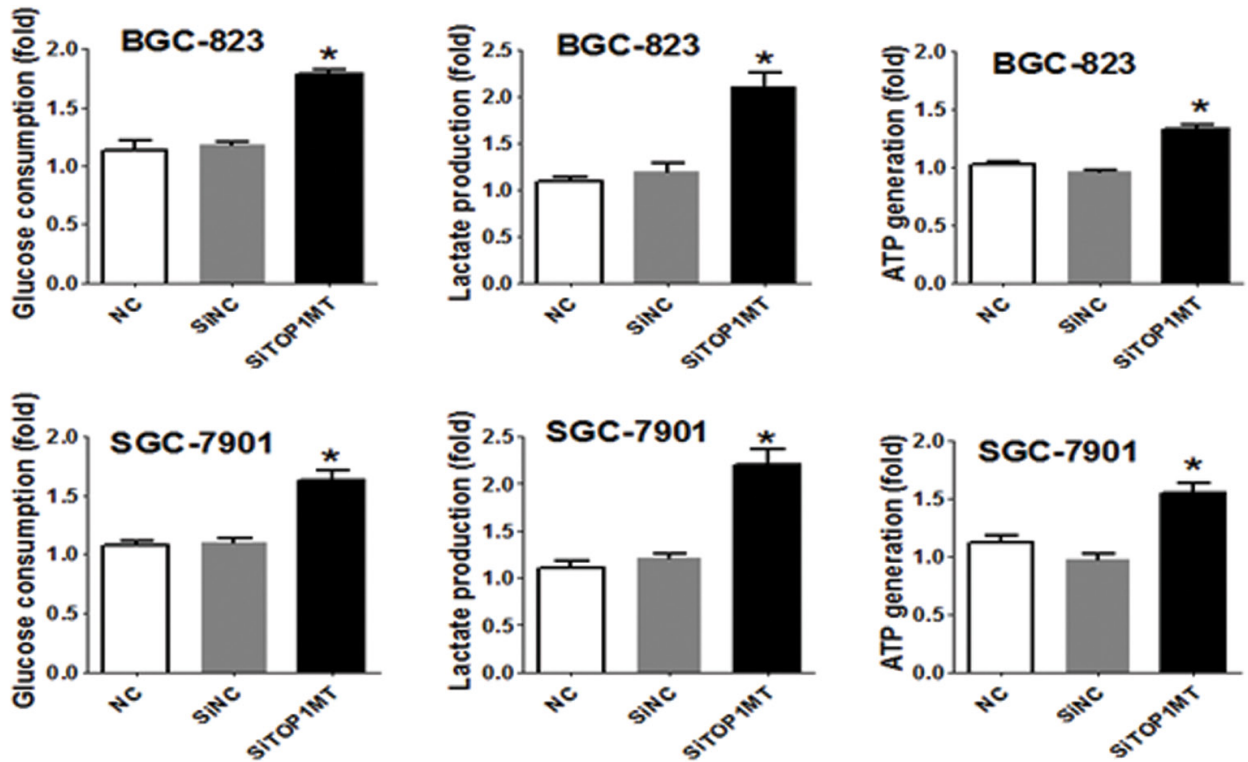

B
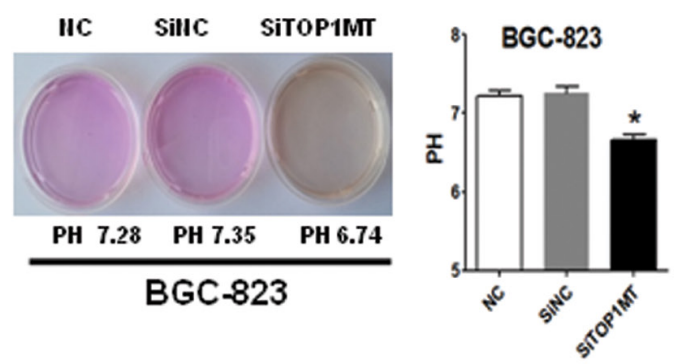

C

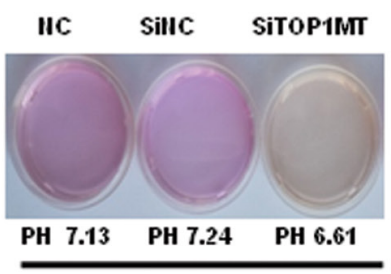

SGC-7901

D

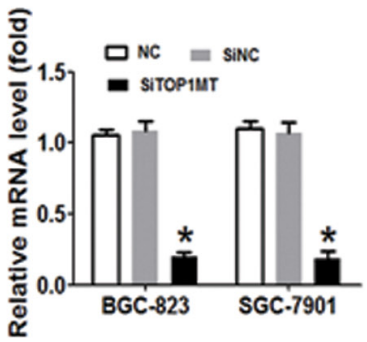

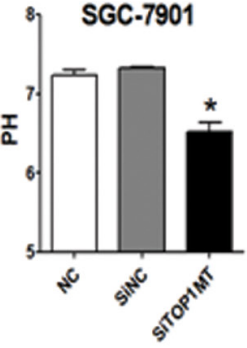

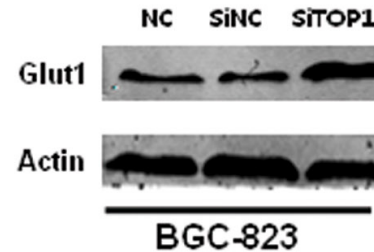

E

$\operatorname{cox}$

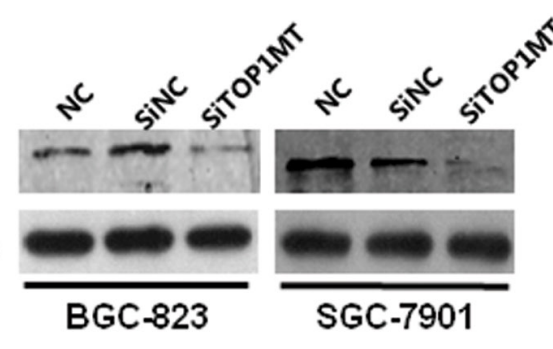

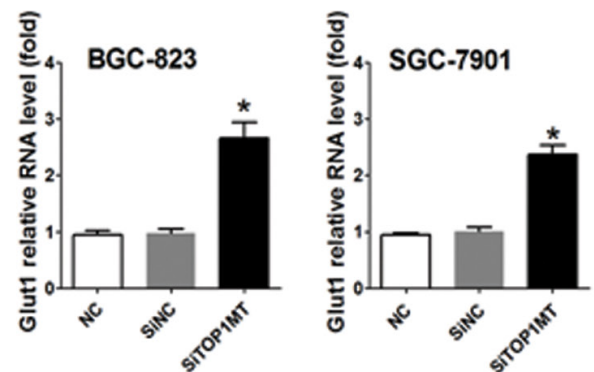

Figure 3

TOP1MT silencing upregulated glucose consumption, lactate production and glucose transporter 1 in GC cells. (A) GC cells of NC, SiNC and SiTOP1MT groups were incubated for $48 \mathrm{~h}$. Glucose uptake, lactate production and ATP generation in different groups of BGC-823 (A, upper) and SGC-7901 (A, lower) cells were measured using enzymatic methods or assay kits. (B) Representative experiments showing pH determination after 48h of culture in NC, SiNC and SiTOP1MT groups. (C) Representative experiments showing glucose transporter 1 (Glut1) mRNA and protein expression, as examined by RT-PCR and Western blotting in NC, SiNC and SiTOP1MT groups. (D and E) Representative experiments showing cytochrome C oxidase (COX) mRNA (D) and protein expression (E), examined by RT-PCR and Western blotting in NC, SiNC and SiTOP1MT groups. ${ }^{*} P<0.05$ in comparisons of the SiTOP1MT-transfected group with the SiNC or NC group. A full colour version of this figure is available at http://dx.doi.org/10.1530/ERC-17-0058. 
ATP generation (Fig. 3A) and decreased the pH (Fig. 3B) of the supernatants in BGC-823 and SGC-7901 cells. Increased glucose transporter 1 (Glut1) expression further verified the upregulation of glucose metabolism in cells with TOP1MT silencing (Fig. 3C). These results indicated that glycolysis was enhanced in GC cells with TOP1MT silencing.

To further explore the effects of TOP1MT

knockdown on mitochondria in GC cells, we detected cytochrome $\mathrm{C}$ oxidase (COX) expression as a marker of OXPHOS activity in mitochondrial respiration (Sotgia et al. 2012). Compared with the control, siTOP1MT significantly decreased the mRNA and protein expression of COX in GC cells (Fig. 3D and E).

\section{Silencing of TOP1MT induced GC cell progression by targeting LDHA}

To further demonstrate the underlying mechanisms through which TOP1MT promoted GC progression, 227 genes related to glycolysis were analyzed by RT-PCR arrays. The results (Supplementary Fig. 4A) showed that LDHA mRNA expression was higher than that of other 227 genes associated with glycolysis and oxidative phosphorylation in BGC-823 cells. Both siLDHA1 and siLDHA2 significantly inhibited LDHA mRNA (Supplementary Fig. 4B) and protein expression (Supplementary Fig. 4C and D). siLDHA1 was selected and further tests were performed to verify LDHA function in GC cell lines. LDHA mRNA and protein expression (Fig. 4A and Supplementary Fig. 4E) and LDHA activation (Fig. 4B) were significantly increased in BGC-823 and SGC-7901 cells with TOP1MT knockdown. Immunohistochemical staining results of subcutaneous xenograft tumors showed that TOP1MT knockdown enhanced LDHA expression (Fig. 4C) in vivo, consistent with the results of the GC cell lines in vitro. Taken together, these results demonstrated that LDHA expression and activation were enhanced in GC cells with TOP1MT silencing in vitro and in vivo.

To determine whether GC cell progression was associated with increased LDHA expression, we exposed cells to oxamate, a well-known inhibitor of LDHA, and siLDHA. Our results showed that sodium oxamate or siLDHA counteracted the increased migration and invasion induced by TOP1MT silencing (Fig. 4D and Supplementary Fig. 4F). The results of wound-closure migration assays further verified these results. The inhibition of LDHA by sodium oxamate or siLDHA counteracted the increased wound recovery induced by
TOP1MT silencing (Fig. 4E). We also found that sodium oxamate or SiLDHA reversed the enhancement of glucose utilization and lactate production and the decrease in $\mathrm{pH}$ values of the supernatants in BGC-823 and SGC-7901 cells induced by TOP1MT silencing (Fig. 4F).

\section{LDHA was associated with changes in EMT markers induced by TOP1MT silencing}

Changes in EMT markers are observed as an early step in the process of tumor metastasis. In order to evaluate changes in EMT markers in GC metastasis induced by TOP1MT silencing in vivo, we examined the expression of vimentin, fibronectin and E-cadherin, which are important EMT markers that are involved in cell migration and invasion. RT-PCR (Fig. 5A) and Western blotting (Fig. 5B) results showed that in both shTOP1MT/BGC-823 and SGC7901 GC cell lines, the expression levels of vimentin and fibronectin were significantly higher, whereas E-cadherin expression was significantly lower than those in negative control cells.

Next, we examined whether LDHA was associated with changes in EMT markers induced by TOP1MT silencing in vitro. We found that TOP1MT silencing upregulated vimentin and fibronectin mRNA and protein and downregulated E-cadherin mRNA and protein. However, inhibition of LDHA by sodium oxamate counteracted these effects in both BGC-823 and SGC-7901 cells (Fig. 5C and D). These results indicated that changes in EMT markers may be involved in GC progression induced by upregulation of LDHA in TOP1MT-deficient cells. However, TOP1MT knockdown did not increase migration and invasion markers of GC cells cultured in galactose medium. Vimentin, fibronectin and E-cadherin expression had no difference between siTOP1MT and the control or oxamate-treated group in GC cells cultured in galactose medium (Supplementary Fig. 5A, B and C). The results further indicated that glycolysis specifically is required for increased cancer cell invasiveness.

\section{TOP1MT was linked with the clinicopathological characteristics of GC}

Both in vitro and in vivo experiments demonstrated that TOP1MT deficiency promoted GC invasion and migration by enhancing LDHA expression. To further confirm this hypothesis in clinical specimens, we assayed tumor specimens from 295 patients with GC. Using immunohistochemical staining, we analyzed

Published by Bioscientifica Ltd. 
A
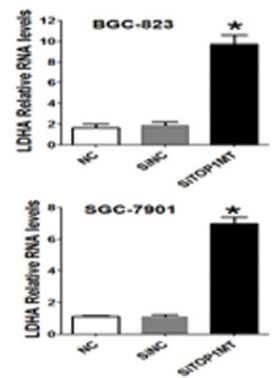

C

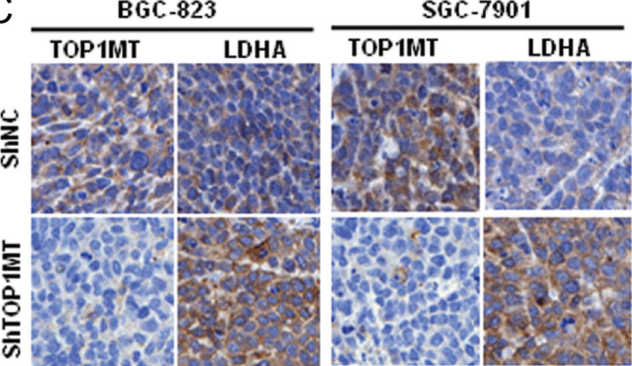

E

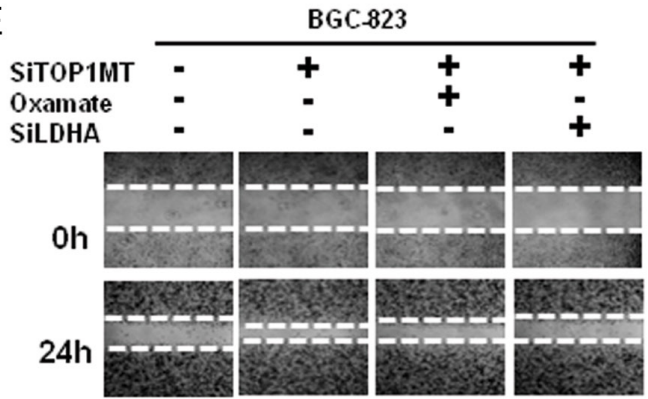

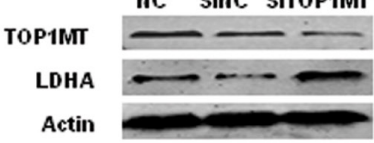

IIC SillC SiTOP1MT

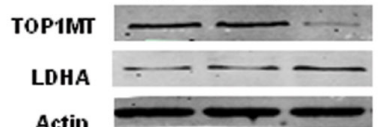

B

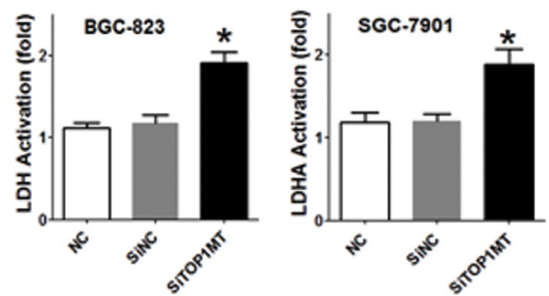

$B$

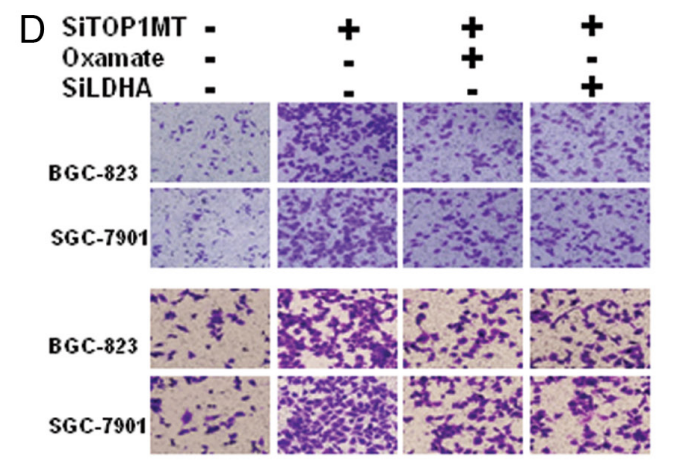

F
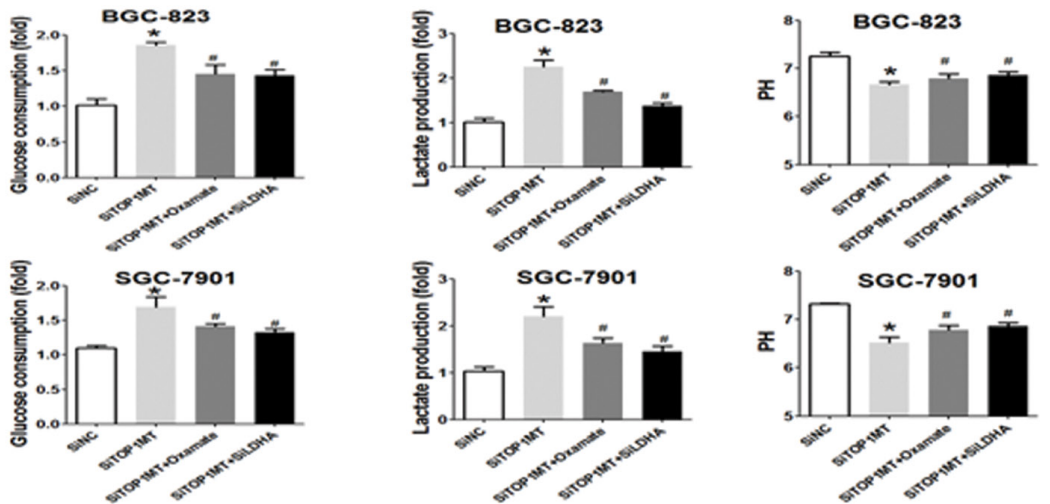

Figure 4

LDHA was required for the upregulation of migration, invasion and glycolysis in GC cells induced by TOP1MT silencing. (A) RT-PCR and Western blot analysis for the detection of LDHA expression in BGC-823 and SGC-7901 cells. (B) LDHA activation was examined for BGC-823 and SGC-7901 cells using LDHA activity assays. (C) Representative images of immunohistochemical staining of LDHA expression for BGC-823 and SGC-7901 cells in subcutaneous xenografts. LDHA expression significantly increased in BGC-823 and SGC-7901 cells with TOP1MT knockdown. (D, E and F) BGC-823 and SGC-7901 cells transfected with SiTOP1MT and/or cultured with LDHA inhibitor (oxamate sodium or siLDHA) were subjected to transwell migration assays (D, upper), Boyden chamber invasion transwell assays $(D$, lower) and wound closure assays (E) to analyze the invasion and migration of GC cells. The results demonstrated LDH inhibition counteracted the migration and invasion induced by TOP1MT knockdown. (F) The results showed that LDHA inhibition reversed the enhancement of glucose consumption (F, left), lactate produced ( $F$, middle) in BGC-823 and SGC-7901 cells induced by TOP1MT silencing, leading to PH values increase (F, right). * $P<0.05$ in comparisons of the SiTOP1MT-transfected group with the control group. ${ }^{P}<0.05$ in comparisons of the SiTOP1MT with oxamate/SiLDHA group with the SiTOP1MT group. A full colour version of this figure is available at http://dx.doi.org/10.1530/ERC-17-0058. 
A

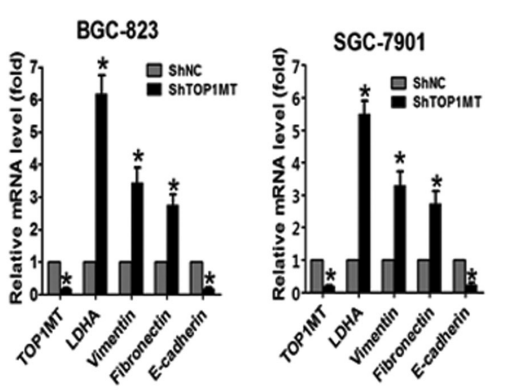

C

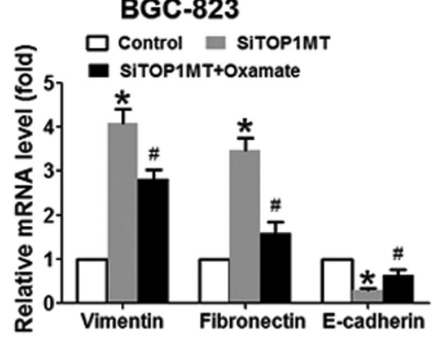

B

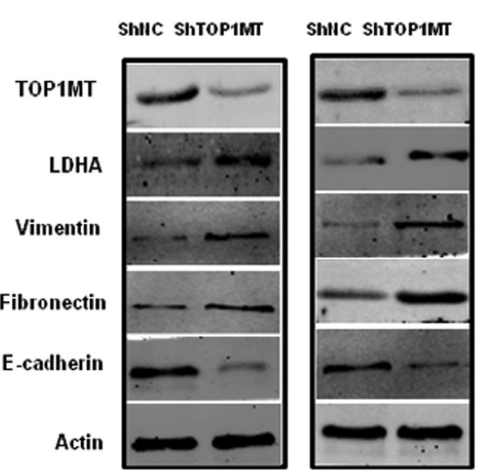

SGC-7901

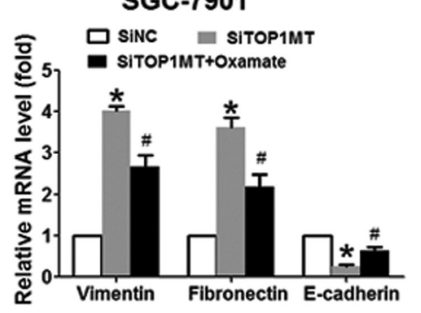

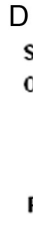

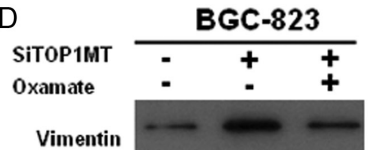

Fibronectin

E-cadherin

Actin

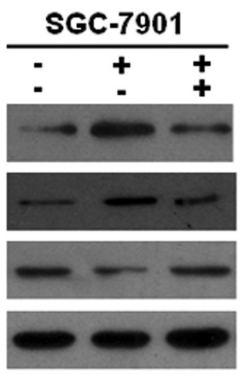

Figure 5

LDHA was associated with changes in EMT markers induced by TOP1MT silencing (A) mRNA and (B) protein expression of vimentin, fibronectin, and E-cadherin in BGC-823 and SGC-7901 cells transfected with shTOP1MT or the scramble control (ShNC) were analyzed by RT-PCR and Western blotting, respectively. (C and D) BGC-823 and SGC-7901 cells infected with SiTOP1MT or inhibited by oxamate sodium were subjected to RT-PCR (C) and Western blotting (D) for detection of vimentin, fibronectin, and E-cadherin mRNA and protein expression, respectively. ${ }^{*}<0.05$ in comparisons of the ShTOP1MT-/SITOP1MT-transfected groups with the ShNC or SiNC group. ${ }^{P}<0.05$ in comparisons of the SiTOP1MT group and the SiTOP1MT with oxamate group.

the relationships between TOP1MT/LDHA expression and clinicopathological features in patients with GC (Supplementary Tables 3 and 4).

TOP1MT expression was significantly decreased in GC specimens relative to normal gastric samples by immunohistochemical staining using monoclonal antiTOP1MT antibodies. Moreover, TOP1MT expression was significantly lower in patients with GC showing lower differentiation, more regional lymph node metastasis, more advanced stage disease and recurrence in stages I-III, and in cases of death in patients with stage IV disease during follow-up (Fig. 6A and Supplementary Fig. 6A). Immunohistochemical staining of LDHA in consecutive sections for these 295 patients with GC showed that LDHA expression was significantly higher in patients with GC having lower differentiation or more regional lymph node metastasis and in cases of death in patients with stage IV disease during follow-up (Fig. 6B and Supplementary Fig. 6B). The disease-free survival rates of patients with stage I-III cancer and the overall survival rates of patients with stage IV cancer were shorter in patients with low TOP1MT expression than those in patients with high TOP1MT expression (Fig. 6C and D).
By analyzing consecutive GC sections, we found that TOP1MT expression was significantly associated with LDHA expression (Fig. 6E and F). These clinical results were consistent with the results of our in vitro and in vivo experiments using GC cell lines.

\section{Discussion}

To the best of our knowledge, this is the first paper to study the function of TOP1MT in GC. We demonstrated the critical role of TOP1MT in promoting GC progression by regulating aerobic glycolysis. To evaluate the role of TOP1MT in enhancing GC invasion and migration and the underlying metabolic mechanism, we provided evidence in two GC cell lines in vitro and in vivo and clinical data for 295 patients with GC. We found that TOP1MT expression was lower in GC tissue specimens and cell lines than that in normal gastric tissues and cell lines. Knockdown of TOP1MT expression promoted the metastasis of GC cells in vitro and in vivo. TOP1MT deficiency significantly increased glucose consumption, lactate production, ATP production and Glut1 expression, indicating that glycolysis was enhanced. In addition, 
A

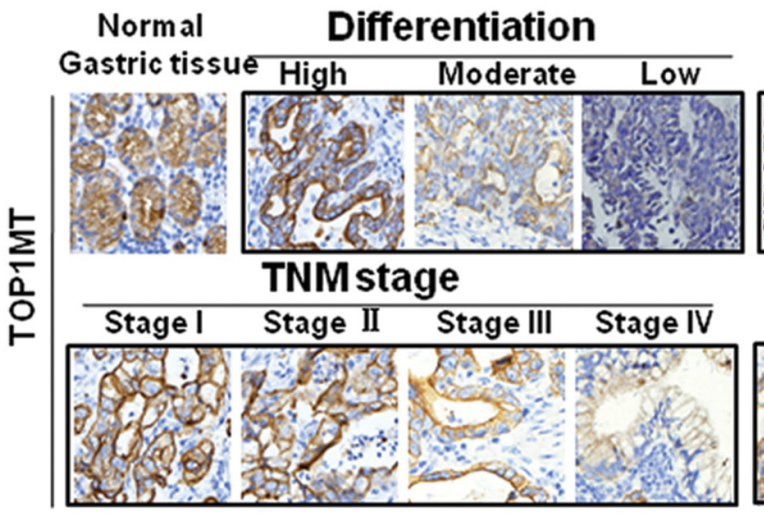

B

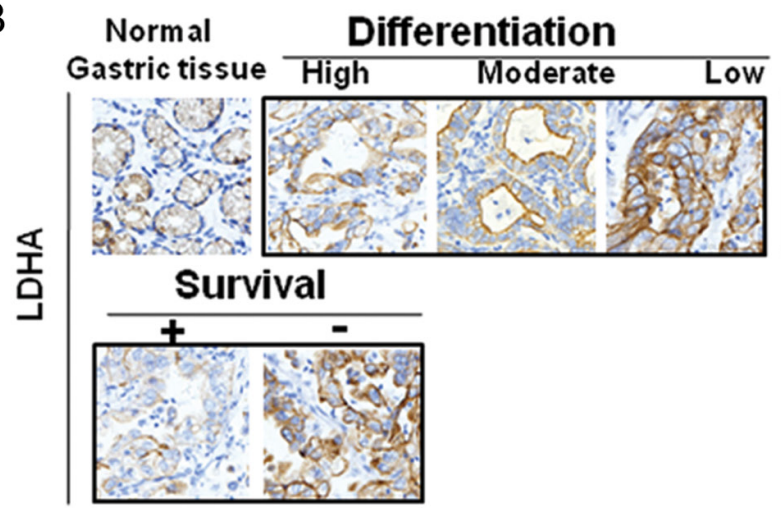

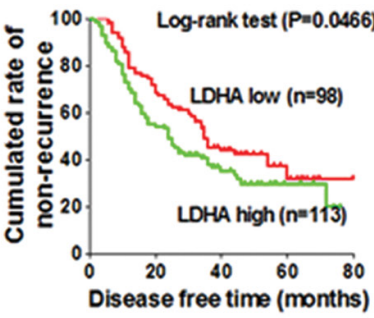

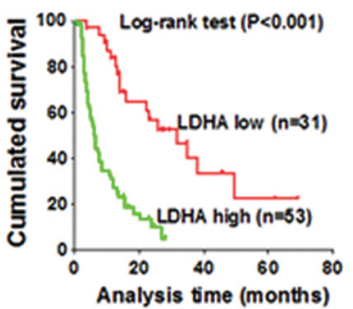

Lymph node
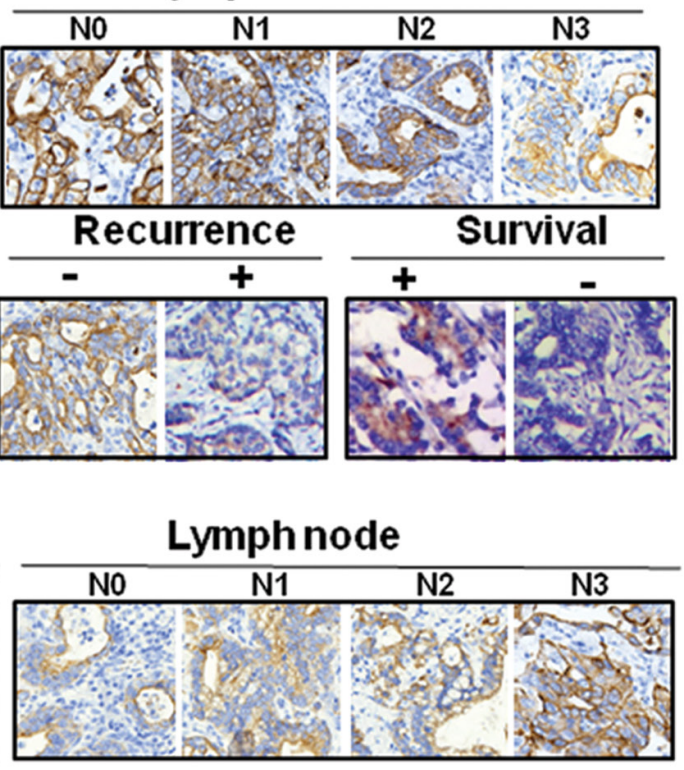

E

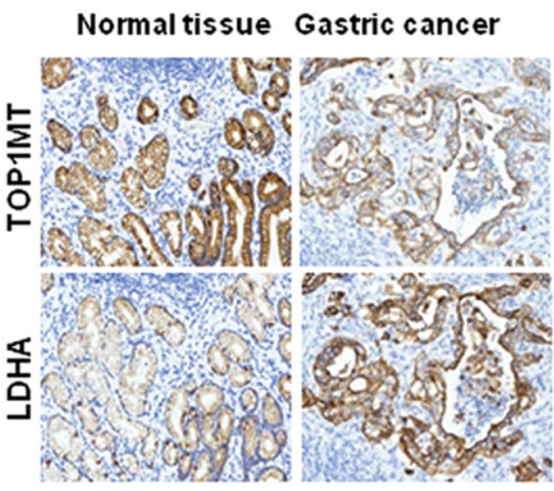

$F$

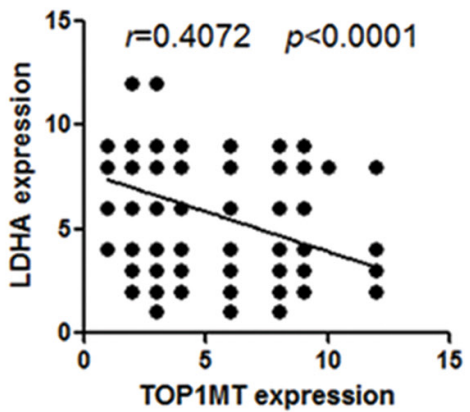

Figure 6

TOP1MT expression in patients with GC and its correlation with the clinicopathological characteristics of GC. Immunohistochemical staining of 295 primary GC specimens for TOP1MT or LDHA protein was performed using paraffin-embedded GC tissue specimens. (A) Representative images of immunohistochemical staining of TOP1MT expression in each group related to differentiation, lymph node metastasis, TNM stage, recurrence, and survival (magnification, 200x). (B) Representative images of immunohistochemical staining of LDHA expression in each group related to differentiation, lymph node metastasis, and survival (magnification, 200x). (C and D) Survival rates of patients with stage IV GC were analyzed by the Kaplan-Meier method. Log-rank tests were used to assess the survival of patients with stage IV GC in relation to TOP1MT (C) or LDHA (D) expression. (E) Representative images of immunohistochemical staining of TOP1MT and LDHA expression in consecutive sections. (F) Direct relationship between TOP1MT and LDHA expression in paraffin-embedded GC tissue specimens $(n=295$; Pearson correlation coefficient, $r=0.4072 ; P<0.0001)$. ${ }^{*}<0.05$. A full colour version of this figure is available at http://dx.doi.org/10.1530/ERC-17-0058.

http://erc.endocrinology-journals.org DOI: 10.1530/ERC-17-0058
C) 2017 The authors Printed in Great Britain
Published by Bioscientifica Ltd 
TOP1MT deficiency significantly increased the mRNA and protein expression levels and activity of LDHA, a key enzyme involved in glycolysis. However, LDHA inhibition counteracted GC invasion and metastasis induced by TOP1MT deficiency. This result suggested that the enhancement of GC invasion and metastasis induced by TOP1MT deficiency was associated with LDHA in GC cells. Upregulation of LDHA in TOP1MTknockdown GC cells changed the EMT markers to promote cell metastasis. In order to further verify the role of TOP1MT in GC, we analyzed the clinical data of 295 patients with GC and found that TOP1MT deficiency was related to the increased tumor metastasis or recurrence and shorter survival time. Therefore, our present study results indicated that TOP1MT may be an underlying target gene regulating tumor metabolism, potentially contributing to the development of novel therapeutic strategies in clinical oncology.

For the first time, we assessed the expression and role of TOP1MT in cancer cells. To clarify its role in GC, cell cycle and cell apoptosis were analyzed by flow cytometry (data not shown), and cell proliferation was determined by MTT and colony-formation assays. Consequently, we found that TOP1MT deficiency did not affect GC cell cycle distribution, apoptosis (data not shown) and proliferation, consistent with previous results (Douarre et al. 2012). Studies have demonstrated that TOP1MT is critical for maintaining normal cellular metabolism in human cells; it can maintain mitochondrial physiological functions and promote OXPHOS to generate cellular ATP. TOP1MT deficiency leads to the dysfunction of mitochondria and enhances glycolysis in normal cells (Douarre et al. 2012). Since TOP1MT is vital for the expression of the OXPHOSrelated genes in mitochondrial DNA, we hypothesize that TOP1MT deficiency may switch cellular glucose metabolism from OXPHOS to glycolysis because of the impaired OXPHOS in cancer cells.

Our results showed that TOP1MT deficiency inhibited COX expression in GC cells, indicating that TOP1MT deficiency was associated with decreased mitochondrial OXPHOS activity. Moreover, TOP1MT knockdown attenuated the expression of COX, a part of complex IV of the electron transport chain. ROS are byproducts of the mitochondrial electron transport chain (Stowe \& Camara 2009). Thus, ROS production decreased because of regulation of the electron transport chain during mitochondrial respiration. Low levels of ROS suppress anoikis of tumor cells, promoting cancer metastasis (Li et al. 1999, Kamarajugadda et al. 2012).
We found that TOP1MT deficiency significantly increased GC cell aerobic glycolysis, also called the Warburg effect. Studies have demonstrated that the Warburg effect is the main pathway of glucose metabolism through which cancer cells generate energy (in the form of ATP) and promote biosynthesis, even under aerobic conditions (Warburg 1956, Gatenby \& Gillies 2004, Semenza 2008). In fact, only 5\% of glucose is metabolized by the OXPHOS pathway in mitochondria in malignant cells (Vander Heiden et al. 2009). TOP1MT deficiency may further inhibit the OXPHOS pathway of glucose metabolism and upregulate the Warburg effect in GC cells. Because cancer cells show rapid proliferation and enhanced invasiveness, oxygen consumption is increased to satisfy the multiple biosynthetic needs of cancer cells. Thus, intratumoral hypoxia is a universal characteristic of rapidly growing tumors. This hypoxic environment promotes HIF-1 activation, which activates its target genes (Kaelin \& Ratcliffe 2008). LDHA is a transcriptional target of HIF-1. Thus, HIF-1 activation induces the expression of LDHA enzyme, which promotes pyruvate-to-lactate conversion and decreases the pyruvate flux into mitochondria. This process may stimulate glycolysis and weaken mitochondrial OXPHOS. The enhanced Warburg effect produces more lactate and thus promotes the formation of an acidic microenvironment. This may represent another mechanism through which the metastasis and invasion of tumor cells are increased (Baumann et al. 2009, Hirschhaeuser et al. 2011, Vegran et al. 2011).

In contrast, the upregulation of TOP1MT in tumor cells may downregulate the Warburg effect, shifting glucose metabolism from glycolysis to oxidative metabolism. This glucose metabolic normalization stimulates the TCA cycle and OXPHOS and increases ROS generation, resulting in inhibition of the progression of certain types of cancer cells (Bonnet et al. 2007). This may be a novel strategy for reversing the malignant biological behaviors of tumors. However, this hypothesis still needs to be explored further.

We confirmed that in TOP1MT-deficient GC cells, the enhanced Warburg effect increased tumor cell invasion and metastasis and promoted the EMT by upregulated LDHA. This result is consistent with previous findings demonstrating that the Warburg effect promotes tumor metastasis (Hsu \& Sabatini 2008, Hirschhaeuser et al. 2011, Chen 2012, Lu et al. 2015). Moreover, previous studies of colorectal cancer (Koukourakis et al. 2006), breast cancer (Wang et al. 2012) and non-small cell-lung cancer (Koukourakis et al. 2003) have demonstrated that LDHA expression and activity are elevated in neoplasms.

Published by Bioscientifica Ltd. 
LDHA is one of the most important enzymes controlling the speed of glycolysis and promoting cancer progression (Le et al. 2010, Zhang et al. 2012, Augoff et al. 2015). However, we found that LDHA expression and activity were further increased in TOP1MT-deficient GC cells. LDHA inhibition significantly counteracted GC invasion and metastasis and the EMT caused by TOP1MT deficiency. This indicated that TOP1MT deficiency promoted the invasion and metastasis of tumor cells mainly through the Warburg effect and that LDHA may have played an important role in this process. Although our present study demonstrated the role of TOP1MT in GC metastasis and the underlying mechanisms responsible for this progression, we still plan to carry out further studies to confirm the role of TOP1MT in GC, including inhibiting LDHA by siRNA or shRNA in vitro and in vivo, which will yield more evidence to support our findings.

In summary, this report was the first to focus on the role of TOP1MT in tumors. We identified a new mechanism underlying TOP1MT-deficient GC metastasis and showed that TOP1MT played an important role in cancer cells. Therefore, upregulation of TOP1MT and application of LDHA inhibitors may inhibit GC metastasis.

\section{Supplementary data}

This is linked to the online version of the paper at http://dx.doi.org/10.1530/ ERC-17-0058.

\section{Declaration of interest}

The authors declare there is no conflict of interest that could be perceived as prejudicing the impartiality of the research reported.

\section{Funding}

This work was supported by the National Natural Science Foundation of China (grant nos. 81502116 and 81472314) and the Special Foundation for National Clinical Specialties of China (to the Department of Oncology, Nanfang Hospital).

\section{Acknowledgements}

The authors thank LetPub (www.letpub.com) for linguistic assistance in writing this manuscript. The authors have no conflicts of interest to disclose.

\section{References}

Augoff K, Hryniewicz-Jankowska A \& Tabola R 2015 Lactate dehydrogenase 5: an old friend and a new hope in the war on cancer. Cancer Letters 358 1-7. (doi:10.1016/j.canlet.2014.12.035)
Baumann F, Leukel P, Doerfelt A, Beier CP, Dettmer K, Oefner PJ, Kastenberger M, Kreutz M, Nickl-Jockschat T, Bogdahn U, et al. 2009 Lactate promotes glioma migration by TGF-beta2-dependent regulation of matrix metalloproteinase-2. Neuro-Oncology 11 368-380. (doi:10.1215/15228517-2008-106)

Bonnet S, Archer SL, Allalunis-Turner J, Haromy A, Beaulieu C, Thompson R, Lee CT, Lopaschuk GD, Puttagunta L, Bonnet S, et al. 2007 A mitochondria-K+ channel axis is suppressed in cancer and its normalization promotes apoptosis and inhibits cancer growth. Cancer Cell 11 37-51. (doi:10.1016/j.ccr.2006.10.020)

Chen EI 2012 Mitochondrial dysfunction and cancer metastasis. Journal of Bioenergetics and Biomembranes 44 619-622. (doi:10.1007/s10863012-9465-9)

DiMauro S \& Schon EA 1998 Nuclear power and mitochondrial disease. Nature Genetics 19 214-215. (doi:10.1038/883)

Douarre C, Sourbier C, Dalla Rosa I, Brata Das B, Redon CE, Zhang H, Neckers L \& Pommier Y 2012 Mitochondrial topoisomerase I is critical for mitochondrial integrity and cellular energy metabolism. PLOS ONE 7 e41094. (doi:10.1371/journal.pone.0041094)

Fantin VR, St-Pierre J \& Leder P 2006 Attenuation of LDH-A expression uncovers a link between glycolysis, mitochondrial physiology, and tumor maintenance. Cancer Cell 9 425-434. (doi:10.1016/j. ccr.2006.04.023)

Fiume L, Manerba M, Vettraino M \& Di Stefano G 2014 Inhibition of lactate dehydrogenase activity as an approach to cancer therapy. Future Medicinal Chemistry 6 429-445. (doi:10.4155/fmc.13.206)

Ganapathy V, Thangaraju M \& Prasad PD 2009 Nutrient transporters in cancer: relevance to Warburg hypothesis and beyond. Pharmacology and Therapeutics 121 29-40. (doi:10.1016/j.pharmthera.2008.09.005)

Gatenby RA \& Gillies RJ 2004 Why do cancers have high aerobic glycolysis? Nature Reviews Cancer 4 891-899. (doi:10.1038/nrc1478)

Giatromanolaki A, Sivridis E, Gatter KC, Turley H, Harris AL, Koukourakis MI \& Tumour and Angiogenesis Research Group 2006 Lactate dehydrogenase 5 (LDH-5) expression in endometrial cancer relates to the activated VEGF/VEGFR2(KDR) pathway and prognosis. Gynecologic Oncology 103 912-918. (doi:10.1016/j.ygyno.2006.05.043)

Goto Y, Hayashi R, Kang D \& Yoshida K 2006 Acute loss of transcription factor E2F1 induces mitochondrial biogenesis in HeLa cells. Journal of Cellular Physiology 209 923-934. (doi:10.1002/jcp.20802)

Hirschhaeuser F, Sattler UG \& Mueller-Klieser W 2011 Lactate: a metabolic key player in cancer. Cancer Research 71 6921-6925. (doi:10.1158/0008-5472.CAN-11-1457)

Hsu PP \& Sabatini DM 2008 Cancer cell metabolism: Warburg and beyond. Cell 134 703-707. (doi:10.1016/j.cell.2008.08.021)

Kaelin WG Jr \& Ratcliffe PJ 2008 Oxygen sensing by metazoans: the central role of the HIF hydroxylase pathway. Molecular Cell $\mathbf{3 0}$ 393-402. (doi:10.1016/j.molcel.2008.04.009)

Kamarajugadda S, Stemboroski L, Cai Q, Simpson NE, Nayak S, Tan M \& Lu J 2012 Glucose oxidation modulates anoikis and tumor metastasis. Molecular and Cellular Biology 32 1893-1907. (doi:10.1128/MCB.06248-11)

Kolev Y, Uetake H, Takagi Y \& Sugihara K 2008 Lactate dehydrogenase-5 (LDH-5) expression in human gastric cancer: association with hypoxia-inducible factor (HIF-1alpha) pathway, angiogenic factors production and poor prognosis. Annals of Surgical Oncology $\mathbf{1 5}$ 2336-2344. (doi:10.1245/s10434-008-9955-5)

Koukourakis MI, Giatromanolaki A, Sivridis E, Bougioukas G, Didilis V, Gatter KC, Harris AL \& Tumour and Angiogenesis Research Group 2003 Lactate dehydrogenase-5 (LDH-5) overexpression in non-smallcell lung cancer tissues is linked to tumour hypoxia, angiogenic factor production and poor prognosis. British Journal of Cancer 89 877-885. (doi:10.1038/sj.bjc.6601205)

Koukourakis MI, Giatromanolaki A, Sivridis E, Gatter KC, Harris AL \& Tumour and Angiogenesis Research Group 2006 Lactate dehydrogenase 5 expression in operable colorectal cancer: strong http://erc.endocrinology-journals.org

DOI: 10.1530/ERC-17-0058
() 2017 The authors Printed in Great Britain
Published by Bioscientifica Ltd 
association with survival and activated vascular endothelial growth factor pathway-a report of the Tumour Angiogenesis Research Group. Journal of Clinical Oncology 24 4301-4308. (doi:10.1200/ JCO.2006.05.9501)

Koukourakis MI, Giatromanolaki A, Winter S, Leek R, Sivridis E \& Harris AL 2009 Lactate dehydrogenase 5 expression in squamous cell head and neck cancer relates to prognosis following radical or postoperative radiotherapy. Oncology 77 285-292. (doi:10.1159/000259260)

Kroemer G \& Pouyssegur J 2008 Tumor cell metabolism: cancer's Achilles' heel. Cancer Cell 13 472-482. (doi:10.1016/j. ccr.2008.05.005)

Le A, Cooper CR, Gouw AM, Dinavahi R, Maitra A, Deck LM, Royer RE, Vander Jagt DL, Semenza GL \& Dang CV 2010 Inhibition of lactate dehydrogenase A induces oxidative stress and inhibits tumor progression. PNAS 107 2037-2042. (doi:10.1073/pnas.0914433107)

Li AE, Ito H, Rovira, II, Kim KS, Takeda K, Yu ZY, Ferrans VJ \& Finkel T 1999 A role for reactive oxygen species in endothelial cell anoikis. Circulation Research 85 304-310. (doi:10.1161/01.RES.85.4.304)

Lin L, Huang H, Liao W, Ma H, Liu J, Wang L, Huang N, Liao Y \& Liao W 2015 MACC1 supports human gastric cancer growth under metabolic stress by enhancing the Warburg effect. Oncogene $\mathbf{3 4}$ 2700-2710. (doi:10.1038/onc.2014.204)

Lu J, Tan M \& Cai Q 2015 The Warburg effect in tumor progression: mitochondrial oxidative metabolism as an anti-metastasis mechanism. Cancer Letters 356 156-164. (doi:10.1016/j.canlet.2014.04.001)

Semenza GL 2008 Tumor metabolism: cancer cells give and take lactate. Journal of Clinical Investigation 118 3835-3837. (doi:10.1172/ JCI37373)

Sotgia F, Whitaker-Menezes D, Martinez-Outschoorn UE, Flomenberg N, Birbe RC, Witkiewicz AK, Howell A, Philp NJ, Pestell RG \& Lisanti MP 2012 Mitochondrial metabolism in cancer metastasis: visualizing tumor cell mitochondria and the 'reverse Warburg effect' in positive lymph node tissue. Cell Cycle 11 1445-1454. (doi:10.4161/cc.19841)

Stowe DF \& Camara AK 2009 Mitochondrial reactive oxygen species production in excitable cells: modulators of mitochondrial and cell function. Antioxidants and Redox Signaling 11 1373-1414. (doi:10.1089/ars.2008.2331)

Vander Heiden MG, Cantley LC \& Thompson CB 2009 Understanding the Warburg effect: the metabolic requirements of cell proliferation Science 324 1029-1033. (doi:10.1126/science.1160809)

Vegran F, Boidot R, Michiels C, Sonveaux P \& Feron O 2011 Lactate influx through the endothelial cell monocarboxylate transporter MCT1 supports an NF-kappaB/IL-8 pathway that drives tumor angiogenesis. Cancer Research 71 2550-2560. (doi:10.1158/00085472.CAN-10-2828)

Wang ZY, Loo TY, Shen JG, Wang N, Wang DM, Yang DP, Mo SL, Guan XY \& Chen JP 2012 LDH-A silencing suppresses breast cancer tumorigenicity through induction of oxidative stress mediated mitochondrial pathway apoptosis. Breast Cancer Research and Treatment 131 791-800. (doi:10.1007/s10549-011-1466-6)

Wang L, Wu Y, Lin L, Liu P, Huang H, Liao W, Zheng D, Zuo Q, Sun L, Huang N, et al. 2013 Metastasis-associated in colon cancer-1 upregulation predicts a poor prognosis of gastric cancer, and promotes tumor cell proliferation and invasion. International Journal of Cancer 133 1419-1430. (doi:10.1002/ijc.28140)

Warburg O 1956 On the origin of cancer cells. Science 123 309-314. (doi:10.1126/science.123.3191.309)

Xia J, Wang H, Huang H, Sun L, Dong S, Huang N, Shi M, Bin J, Liao Y \& Liao W 2016 Elevated orai1 and STIM1 expressions upregulate MACC1 expression to promote tumor cell proliferation, metabolism, migration, and invasion in human gastric cancer. Cancer Letters $\mathbf{3 8 1}$ 31-40. (doi:10.1073/pnas.191321998)

Zhang H, Barcelo JM, Lee B, Kohlhagen G, Zimonjic DB, Popescu NC \& Pommier Y 2001 Human mitochondrial topoisomerase I. PNAS 98 10608-10613. (doi:10.1073/pnas.191321998)

Zhang Y, Zhang X, Wang X, Gan L, Yu G, Chen Y, Liu K, Li P, Pan J, Wang J, et al. 2012 Inhibition of LDH-A by lentivirus-mediated small interfering RNA suppresses intestinal-type gastric cancer tumorigenicity through the downregulation of Oct4. Cancer Letters 321 45-54. (doi:10.1016/j.canlet.2012.03.013)

Received in final form 26 August 2017

Accepted 4 September 2017

Accepted Preprint published online 5 September 2017
() 2017 The authors Printed in Great Britain
Published by Bioscientifica Ltd. 